Artículo

\title{
Fertilización NPK, distribución de biomasa y número de minitubérculos de papa en invernadero
}

\author{
Román Flores-López ${ }^{\S}$ \\ Maricela Casimiro-Marín \\ Erasto Sotelo-Ruiz \\ Oswaldo Rubio-Covarrubias \\ Humberto López-Delgado
}

${ }^{1}$ Sitio Experimental Metepec-INIFAP. Carretera Toluca-Zitácuaro km 4.5, Vialidad Adolfo López Mateos, Col. San José Barbabosa, Zinacantepec, Estado de México. CP. 51350. (mary_marin_c@ hotmail.com; sotelo.erasto@inifap.gob.mx; rubio.oswaldo@inifap.gob.mx; humlopde@hotmail.com).

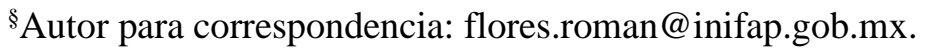

\section{Resumen}

El objetivo de esta investigación fue evaluar la respuesta de la papa (Solanum tuberosum) a la fertilización de nitrógeno $(\mathrm{N})$, fósforo $(\mathrm{P})$ y potasio $(\mathrm{K})$, en la acumulación de biomasa, índice de cosecha y número de tubérculos bajo condiciones de hidroponía bajo invernadero. El diseño experimental utilizado fue el San Cristóbal, con 12 tratamientos. Las variables que se evaluaron fueron: biomasa de hoja, tallo, raíz, estolón y tubérculo, índice de cosecha y número de tubérculos. La distribución de biomasa en todos los tratamientos, con respecto a la biomasa total, varió de 9.88 a $13.1 \%$ en hoja; 1.83 a $4 \%$ tallo, 1.9 a $4.9 \%$ raíz, 0.8 a $1.31 \%$ estolón y de 77.6 a $83.6 \%$ en tubérculo. El tratamiento de fertilización con $250 \mathrm{~N}-80 \mathrm{P}-300 \mathrm{~K} \mathrm{mg} \mathrm{L} \mathrm{K}^{-1}$ obtuvo la mayor acumulación de biomasa total por planta, $63.54 \mathrm{~g}$; para hoja, $6.78 \mathrm{~g}$, tallo $2.36 \mathrm{~g}$, estolón 0.5 , raíz $1.2 \mathrm{~g}$ y tubérculos $52.56 \mathrm{~g}$. Mientras el tratamiento que presentó el índice más alto de cosecha fue T2 (200N-30P-250K) con 0.83 , sólo diferente a los tratamientos T7(100N; 130P; 350K) y T11(150N; 180P; 300K) con 0.77 y 0.78 , respectivamente. El índice de cosecha no estuvo relacionado con el número de tubérculos por planta, pues los tratamientos con mayor número de estos fueron el T8 (200N-130P-250K) con 18.6 tubérculos y T12(150N-80P-400K) 18.2, con un índice de cosecha de 0.82 , mientras el T3 con el mayor indice (0.83) solamente produjo 13.7 tubérculos por planta.

Palabras clave: biomasa, hidroponía, papa.

Recibido: octubre de 2020

Aceptado: diciembre de 2020 


\section{Introducción}

La papa (Solanum tuberosum L.) demanda grandes cantidades de nutrientes, que son importantes en el rendimiento y la calidad de los tubérculos (Morales et al., 2013), principalmente nitrógeno $(\mathrm{N})$, fósforo (P) y potasio (K) durante todo su ciclo (White et al., 2007). Bertsch (2003) menciona que absorbe 220, 20, 240, 60 y $20 \mathrm{~kg} \mathrm{ha}^{-1}$ de $\mathrm{N}, \mathrm{P}, \mathrm{K}, \mathrm{Ca}$ y $\mathrm{Mg}$, respectivamente, para una producción de $20 \mathrm{t} \mathrm{ha}^{-1}$; sin embargo, Rocha y Quijano (2015) mencionan el uso de una fórmula de fertilización de 200-300-200 de NPK, más $30 \mathrm{~kg}$ de sulfato de zinc y $25 \mathrm{~kg}$ de boro con rendimientos superiores a las $40 \mathrm{t} \mathrm{ha}^{-1}$, lo que evidencia los altos requerimientos nutrimentales que presenta el cultivo.

En invernadero se cultiva en sustratos orgánicos (turba, fibra de coco, cascarilla de arroz) mezclado con sustratos inorgánicos como perlita o alguna otra grava de diámetro pequeño; sin embargo, la fertilización se hace empleando mezclas físicas de fertilizantes granulados o bien fórmulas de fertilizantes solubles o soluciones nutritivas hidropónicas (Flores et al., 2009). La mayor parte del nitrógeno $(\mathrm{N})$ absorbido por la planta ocurre durante la etapa de crecimiento vegetativo y antes de la etapa de llenado del tubérculo consume más de $50 \%$ con una demanda diaria de $7 \mathrm{~kg} \mathrm{ha}^{-1} \mathrm{día}^{-1}$. Para el caso del fósforo $(\mathrm{P})$ la demanda fluctúa entre 0.4 a $0.9 \mathrm{~kg} \mathrm{ha}^{-1}$ día $^{-1}$, a mitad del ciclo dependiendo de la variedad y clima. En relación con el potasio (K) la absorción es de 5 a 14 kg ha-1 día ${ }^{-1}$ (Horneck y Rosen, 2008).

El exceso o deficiencia de $\mathrm{N}$ tienen consecuencias en el rendimiento de tubérculos (Westermann, 2005). El N favorece el desarrollo foliar, lográndose aumentar la superficie de fotosíntesis, lo que conlleva a la producción de almidón, incide directamente en la translocación del almidón desde las hojas hacia los tubérculos, influye en el rendimiento, la altura de la planta, número de tubérculos por unidad de área, porcentaje de proteínas y materia seca (Ramírez et al., 2004). El exceso de nitrógeno puede alargar el ciclo del cultivo, afectar el rendimiento de manera negativa e incrementar los riesgos de enfermedades foliares (Sierra et al., 2002).

Mientras, el fósforo influye en el crecimiento, acelera la madurez, mejora la calidad y el rendimiento; ayuda a la división y crecimiento celular (Valverde et al., 1998). En las plantas de papa con deficiencia de $\mathrm{P}$ se retarda el crecimiento apical, dando lugar a plantas pequeñas y rígidas y reduce la formación de almidón en los tubérculos que se manifiesta con manchas necróticas distribuidas en el tubérculo (Pumisacho y Sherwood, 2002). Por otro lado, es de dominio generalizado que el potasio actúa como activador de enzimas esenciales en fotosíntesis, respiración, formación de almidón y proteínas.

Después del nitrógeno, el potasio es el nutriente mineral requerido en mayor cantidad por las plantas de papa, es muy móvil dentro de ella y es absorbido del suelo como catión K+ (Becerra y Nústez, 2007). Los síntomas de deficiencia aparecen primero en las hojas de mayor edad, las plantas quedan pequeñas por el acortamiento de los entrenudos, los tallos y ramas son débiles, las raíces tienen pobre desarrollo, los estolones quedan cortos y se producen tubérculos pequeños.

La hidroponía es una alternativa para la producción de semilla de papa con ventajas sobre el cultivo tradicional, ya que se usa una menor área de cultivo, debido a la mayor densidad por unidad de superficie que se puede manejar (Chuquillanqui et al., 2010). Se ha demostrado que es posible 
aumentar el número de tubérculos producidos mediante la técnica de producción hidropónica (Rolot y Seutin, 1999). Y un requisito para ello es lograr el equilibrio entre las proporciones de nutrientes dentro de la solución nutritiva, la conductividad eléctrica $(\mathrm{CE})$ y $\mathrm{pH}$ según sea necesario para la producción de papa (Chang et al., 2011).

El rendimiento en un cultivo es el resultado final de los procesos de acumulación y distribución de biomasa y está dado por la capacidad del genotipo de acumular biomasa en los órganos de interés económico, por lo que el incremento proporcional de la biomasa destinada a estos órganos garantiza el incremento del rendimiento.

La acumulación y distribución de biomasa en los vegetales son características genotípicas fácilmente afectadas por el ambiente y su interacción (Rajwade et al., 2000). Así, la proporción de biomasa asignada a hojas, tallos y tubérculos en cada momento del desarrollo depende de la cinética de crecimiento y la tasa de distribución, que están gobernadas por el área foliar, clima y disponibilidad de nutrimentos (Heemst, 1986).

La papa (Solanum tuberosum) acumula mayor cantidad de materia seca en los órganos aéreos y las raíces durante la mitad inicial del ciclo biológico y posteriormente disminuye parte de ésta debido a la pérdida de hojas y translocación de fotosintatos hacia los tubérculos (Aguilar et al., 2001), por lo que en determinado momento se da una relación de competencia entre el dosel y los tubérculos (Susnoschi y Shimshi, 1985). Se menciona que el crecimiento de los tubérculos está determinado por las tasas de acumulación y distribución de biomasa en los diferentes órganos de la planta (Khurana y Pandita, 1994) y en ambientes favorables, más de $90 \%$ de los fotosintatos producidos en las hojas son traslocados hacia los tubérculos (Wolf et al., 1990).

El índice de cosecha (IC) determina la relación en distribución de biomasa en la planta completa y los órganos de importancia antropocéntrica, como son los tubérculos, por lo que es considerado como índice de eficiencia fisiologíca (Mora et al., 2005) y en papa, puede variar entre 57 y $91 \%$ cuando los tubérculos han madurado y el follaje está en senescencia (Rajwade et al., 2000) este rango representa las diferencias debidas al ambiente de producción, al genotipo o a la interacción entre ambos factores (Jefferies y Mackerron, 1993), en genotipos precoces de papa el IC es mayor que en los tardíos; en ambos, es afectado por factores ambientales (Sierra, 2002).

En condiciones de invernadero se puede incidir en varios factores que afectan el rendimiento como el genotipo, fecha de siembra, densidad, manejo agronómico, disponibilidad de agua, control de plagas, enfermedades y nutrición del cultivo, siendo esta, el factor más importante en el desarrollo y rendimiento del cultivo de papa. El objetivo de la investigación fue evaluar la respuesta de la papa $(S$. tuberosum) a la fertilización de nitrógeno $(\mathrm{N})$, fósforo $(\mathrm{P})$ y potasio $(\mathrm{K})$ en la acumulación de biomasa, índice de cosecha y rendimiento en condiciones de hidroponía bajo invernadero.

\section{Materiales y métodos}

El experimento se realizó en invernaderos del Instituto Nacional de Investigaciones Forestales, Agrícolas y Pecuarias (INIFAP), Sitio experimental Metepec, ubicado en el km 4.5 carretera Toluca-Zitácuaro, Zinacantepec, Estado de México a $19^{\circ} 17^{\prime} 21^{\prime \prime}$ ' de latitud norte y $99^{\circ} 42^{\prime}$ 49'" de longitud oeste a $2640 \mathrm{msnm}$. La temperatura media dentro del invernadero fue de $15.5^{\circ} \mathrm{C}$ con 
máximas de 36 y mínimas de -0.9. La variedad de papa utilizada en esta investigación fue Nevada, de reciente liberación por el INIFAP, con alto rendimiento, calidad para la industria y tolerancia al manchado interno del tubérculo ocasionado por el síndrome de punta morada de la papa y tolerante a tizón y punta morada.

Se emplearon macetas de $1.8 \mathrm{~L}$ de volumen con perlita grado hortícola de 1 a $4 \mathrm{~mm}$ de diámetro. Se utilizaron minitubérculos de 10 a $15 \mathrm{~mm}$ de diámetrolibres de virus, con un solo brote, los tubérculos fueron tratados con un enraizador (Miyaraiz) a $5 \mathrm{ml} \mathrm{L}^{-1}$ y Miyafungi TH (Trichoderma harsianum), $1 \mathrm{~g} \mathrm{~L}^{-1}$ por inmersión durante 5 minutos.

El riego se hizo con el uso de goteros de $8 \mathrm{~L} \mathrm{~h}^{-1}$ con distribuidor de cuatro salidas. Se programaron cuatro riegos las primeras dos semanas, seguido de cinco las siguientes cuatro semanas y siete las últimas seis, el gasto fue de $33 \mathrm{ml}$ por maceta en cada riego para un gasto máximo de $231 \mathrm{ml}$ por maceta. Los niveles N P K fueron cuatro, distribuidos de acuerdo con el diseño San Cristóbal (Martínez, 1996), 12 tratamientos, cuatro repeticiones y 16 plantas por cada repetición.

Las concentraciones en $\mathrm{mg} \mathrm{L}^{-1}$ fueron de 100, 150, 200 y 250 de nitrógeno; 30, 80, 130 y 180 para fósforo y de 250, 300, 350 y 400 de potasio. Los 12 tratamientos resultantes presentan las siguientes combinaciones de NPK: T1 (100-30-250); T2 (200-30-250); T3 (100-130-250); T4 (200-130-250); T5 (100-30-350); T6 (200-30-350); T7 (100-130-350); T8 (200-130-350); T9 (150-80-300); T10 (250-80-300); T11 (150-180-300) y T12 (150-80-400). Para ello se hicieron las 12 soluciones nutritivas complementadas con $45 \mathrm{mg} \mathrm{L}^{-1}$ de $\mathrm{Mg}, 200 \mathrm{mg} \mathrm{L}^{-1} \mathrm{Ca}, 3 \mathrm{mg} \mathrm{L}^{-1} \mathrm{Fe}-\mathrm{EDTA}, 0.5 \mathrm{mg} \mathrm{L}^{-1}$ $\mathrm{Zn}, 0.5 \mathrm{mg} \mathrm{L}^{-1} \mathrm{Cu}, 0.5 \mathrm{mg} \mathrm{L}^{-1} \mathrm{~B}$. El pH se ajustó a 6 y la conductividad varió de 2 a $2.6 \mathrm{dS} \mathrm{m} \mathrm{m}^{-1}$.

Las variables evaluadas fueron biomasa de hoja, tallo, raíz, estolón y tubérculo, índice de cosecha y número total de mini tubérculos, tomando esta última variable como rendimiento. Para la biomasa se tomaron tres muestras de cada tratamiento y repetición. Cada una de las partes se colocó en bolsas de papel, el secado de estos órganos se hizo en una estufa con circulación de aire forzado a $70{ }^{\circ} \mathrm{C}$ hasta peso constante. Se realizó un Anova, además de una prueba Tukey al $95 \%$ de confianza, utilizando el programa SAS V9.

\section{Resultados y discusión}

La distribución de biomasa en porcentaje siguió el siguiente comportamiento con valores para hoja de 9.88 a $13.1 \%$, tallo de 1.83 a 4\%, raíz 1.9 a 4.9\%, estolón de 0.8 a $1.31 \%$ y tubérculo de 77.6 a $83 \%$ con respecto a la biomasa total de la planta, lo que concuerda con lo mencionado por Alva et al. (2002), quienes mencionan que el porcentaje de biomasa en tuberculo es de 76 al 85\%, en tallo de 3 a $11 \%$ y en hoja de 9 al $13 \%$ en condiciones de campo.

En la Figura 1, se observa la distribución de biomasa promedio de los doce tratamientos de NPK en la variedad Nevada en condiciones de invernadero e hidroponia, donde se aprecia que la biomasa de tubérculo supera la de las demás partes de la planta en porcentaje, tubérculo 82, mientras para hoja es de 11.1, tallo 2.95, raíz, 2.95 y estolones 1.02. Sin embargo, entre tratamientos si se presentaron diferencias estadísticas significativas en biomasa en todos los órganos en estudio (Cuadro1). 


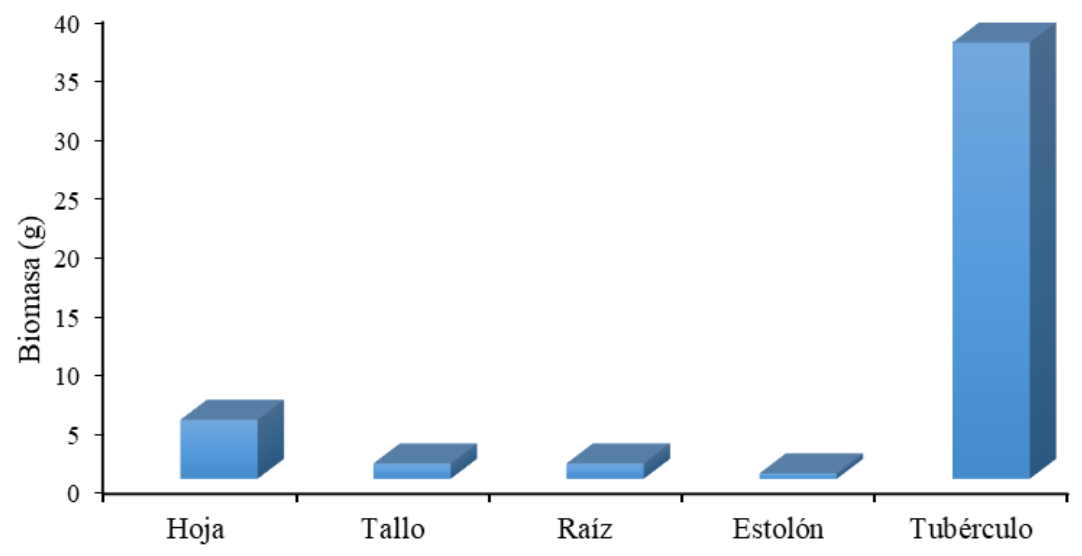

Figura 1. Distribución promedio de biomasa seca de hoja, tallo, raíz, estolón y tubérculo en la variedad Nevada y NPK en hidroponía e invernadero.

Cuadro 1. Acumulación de biomasa de hoja (g materia seca por planta), tallo, raíz, estolón y tubérculo en papa con doce tratamientos de NPK.

\begin{tabular}{cccccc}
\hline Tratamientos & Hoja & Tallo & Raíz & Estolón & Tubérculo \\
\hline T1(100N-30P-250K) & $4.7 \mathrm{bcd}$ & $0.8 \mathrm{fe}$ & $1.2 \mathrm{ba}$ & $0.5 \mathrm{ba}$ & $36.5 \mathrm{~cd}$ \\
T2(200N-30P-250K) & $5.1 \mathrm{bc}$ & $1 \mathrm{fecd}$ & $1.1 \mathrm{~b}$ & $0.6 \mathrm{ba}$ & $40.8 \mathrm{bc}$ \\
T3(100N-130P-250K) & $5.3 \mathrm{bc}$ & $0.9 \mathrm{fed}$ & $1.4 \mathrm{ba}$ & $0.4 \mathrm{bac}$ & $39.1 \mathrm{bcd}$ \\
T4(200N-130P-250K) & $4.2 \mathrm{~cd}$ & $0.7 \mathrm{f}$ & $1.1 \mathrm{~b}$ & $0.4 \mathrm{bac}$ & $25.9 \mathrm{e}$ \\
T5(100N-30P-350K) & $3.8 \mathrm{~d}$ & $0.8 \mathrm{fe}$ & $1.2 \mathrm{ba}$ & $0.3 \mathrm{c}$ & $31.2 \mathrm{ecd}$ \\
T6(200N-30P-350K) & $5.8 \mathrm{ab}$ & $1.7 \mathrm{ba}$ & $1.3 \mathrm{ba}$ & $0.6 \mathrm{a}$ & $48.3 \mathrm{ba}$ \\
T7(100N-130P-350K) & $4.2 \mathrm{~cd}$ & $1.4 \mathrm{becd}$ & $1.7 \mathrm{a}$ & $0.4 \mathrm{bc}$ & $26.7 \mathrm{e}$ \\
T8(200N-130P-250K) & $4.8 \mathrm{bcd}$ & $1.5 \mathrm{bcd}$ & $1.5 \mathrm{ba}$ & $0.5 \mathrm{bac}$ & $40.3 \mathrm{bcd}$ \\
T9(150N-80P-300K) & $5 \mathrm{bcd}$ & $1.4 \mathrm{becd}$ & $1.3 \mathrm{ba}$ & $0.4 \mathrm{bac}$ & $34.5 \mathrm{ecd}$ \\
T10(250N-80P-300K) & $6.7 \mathrm{a}$ & $2.3 \mathrm{a}$ & $1.2 \mathrm{ba}$ & $0.5 \mathrm{bac}$ & $52.5 \mathrm{a}$ \\
T11(150N-180P-300K) & $5.2 \mathrm{bc}$ & $1.6 \mathrm{bc}$ & $1.5 \mathrm{ba}$ & $0.4 \mathrm{bac}$ & $31 \mathrm{ed}$ \\
T12(150N-80P-400K) & $5.2 \mathrm{bc}$ & $1.5 \mathrm{bcd}$ & $1.5 \mathrm{ba}$ & $0.6 \mathrm{a}$ & $37.1 \mathrm{~cd}$ \\
DMS & 1.27 & 0.63 & 0.5 & 0.21 & 8.56 \\
\hline
\end{tabular}

Tratamientos con la misma letra no difieren estadísticamente según la prueba de Tukey $p<0.05$.

La biomasa de hoja representó entre el 9.88 y $13.1 \%$, respecto biomasa de la planta completa, siendo los tratamientos T10 (250N-80P-300K) y T6 (200N-30P-350K) los que presentaron los valores más altos, de 6.7 y 5.8 g, respectivamente (Cuadro1); sin embargo, en porcentaje no es el mayor, para estos tratamientos con 10.5 y $10 \%$ respectivamente, lo que se puede deber al mayor peso seco de tubérculo alcanzado en estos, mientras el menor peso seco de hoja fue para los tratamientos T7 (100N-130P-350K), T4 (200N-130P-250K) y T5 (100N-30P-350K), con $4.2 \mathrm{~g}$ para los dos primeros y $3.8 \mathrm{~g}$ para el último, que representaron porcentajes de $12.21,13$ y $10.19 \%$, mientras la biomasa del tallo, representó entre el 1.8 y $4 \%$ de la biomasa total y el mejor tratamiento 
para esta variable en acumulación de materia seca fue el T 10 con 2.3 g, seguido del T6 1.7 g, con porcentajes de 3.6 y 2.95 y los de menor acumulación de masa seca en tallo fueron los tratamientos T1 (100N-30P-250K) y T5 (100N-30P-350K) con 0.8 g que represento el 1.83 y $2.14 \%$, respectivamente (Cuadro 1). En cuanto a hoja, resultados similares encontró en su estudio Alva et al. (2002), en donde el peso seco de hojas representó de 9 a 13\%, no así para tallo donde menciona que va de 3 a $11 \%$.

En particular los mejores tratamientos tanto en biomasa de hoja como de tallo fueron aquellos con dosis altas de nitrógeno (200 y $\left.250 \mathrm{mg} \mathrm{L}^{-1}\right)$ a diferencia de los tratamientos que contenían la dosis mínima de $\mathrm{N}$ que fue $100 \mathrm{mg} \mathrm{L}^{-1}$, debido a que no obtuvieron un buen desarrollo de follaje y por tanto una menor área de captación de luz. En papa, la fase lineal del crecimiento de los tubérculos puede prolongarse hasta que el índice de área foliar disminuye a casi 1, mientras que la raíz crece rápidamente sólo hasta después de la mitad del ciclo biológico y luego disminuye a los 80 días después de la emergencia, lo que coincide con la pérdida de biomasa en el follaje (Aguilar et al., 2001). Esto es importante considerar, puesto que la toma de muestra se hizo al final del ciclo y pudo haber pérdida de hojas por senescencia.

Los resultados de biomasa de hoja concuerdan con lo mencionado por Zebarth y Ros (2007), quienes señalan que una adecuada aportación de nitrógeno es requerida para mejorar la capacidad del dosel en la intercepción de luz, debido a la participación critica del nitrógeno en el desarrollo vegetativo y acumulación de biomasa. Por otro lado, Coraspe et al. (2008), mencionan que la acumulación de $\mathrm{N}$ en los tubérculos aumentó con las dosis de $\mathrm{N}$ variando de 252.31 a $355.82 \mathrm{mg}$ planta $^{-1}$, mientras que en la parte aérea la variación fue menor, de 103.92 a $172.11 \mathrm{mg}_{\text {planta }}{ }^{-1}$.

La biomasa de raíz representó del 1.9 a $4.9 \%$ con respecto al peso total de la planta; los tratamientos más altos de biomasa de raíz fueron el T7 $(100 \mathrm{~N} ; 130 \mathrm{P} ; 350 \mathrm{~K})$, T11 (150 N; $180 \mathrm{P} ; 300 \mathrm{~K})$ y T12 (150 N; 80 P; $400 \mathrm{~K})$ con 1.7, 1.5 y 1.5 g respectivamente y los más bajos fueron el T4 (200 $\mathrm{N} ; 130 \mathrm{P} ; 250 \mathrm{~K})$ y el T2 $(200 \mathrm{~N} ; 30 \mathrm{P} ; 250 \mathrm{~K})$ con $1.1 \mathrm{~g}$, los únicos tratamientos diferentes estadísticamente entre sí fueron el T7 y T2, siendo el resto de los tratamientos iguales, aún con estos dos tratamientos contrastantes, en general no se encontraron diferencias entre los diferentes niveles de fósforo empleados de 30, 80, 130 y $180 \mathrm{mg} \mathrm{L}^{-1}$ (Cuadro 1), por lo que se considera que se obtuvo buena cantidad de raíz con todos los niveles empleados de fósforo; sin embargo, se puede afirmar que a medida que se incrementan las dosis de fósforo disminuye su aprovechamiento; esto concuerda con lo mencionado por Camozzi (2013).

La biomasa de estolón representó entre 0.79 a $1.31 \%$ de la biomasa total de la planta; los tratamientos con valores más altos fueron el T6 $(200 \mathrm{~N} ; 30 \mathrm{P} ; 350 \mathrm{~K}), \mathrm{T} 12(150 \mathrm{~N} ; 80 \mathrm{P} ; 400 \mathrm{~K}) \mathrm{y}$ el T2 $(100 \mathrm{~N} ; 30 \mathrm{P} ; 350 \mathrm{~K})$ con $0.6 \mathrm{~g}$, mientras el más bajo fue el T5 $(100 \mathrm{~N} ; 30 \mathrm{P} ; 350 \mathrm{~K})$ con 0.3 $\mathrm{g}$, en esta variable con relación a los niveles de fósforo no tuvo mayor efecto, pues los tratamientos con mayor peso tenían dosis de 30 y $80 \mathrm{mg} \mathrm{L}^{-1}$ respectivamente, el T5 (100 N; $\left.30 \mathrm{P} ; 350 \mathrm{~K}\right)$ que mostró bajos valores tenía la misma cantidad de P que el T6 (200 N; 30 P; 350 K) y T7 (100 N; $130 \mathrm{P} ; 350 \mathrm{~K}$, este último con $130 \mathrm{mg} \mathrm{L}^{-1}$ de fósforo, por lo que podemos mencionar que no afecto la biomasa de estolones.

En relación a la biomasa de tubérculo, es importante mencionar que los tratamientos con valores más altos en peso seco de tubérculo, fueron los tratamientos T10 $(250 \mathrm{~N}-80 \mathrm{P}-300 \mathrm{~K})$ y T6 $(200 \mathrm{~N}$; $30 \mathrm{P} ; 350 \mathrm{~K})$ con 52.5 y $48.3 \mathrm{~g}$, respectivamente, con concentraciones de 300 y $350 \mathrm{mg} \mathrm{L}^{-1}$ de 
potasio como se muestra en la Figura 2 y los de menor biomasa de tubérculo, fueron los tratamientos $\mathrm{T} 7$ y T4, el primero con $350 \mathrm{mg} \mathrm{L}^{-1}$ de potasio y con solo $100 \mathrm{mg} \mathrm{L}^{-1}$ de nitrógeno, lo anterior indica la importancia del uso de altas concentraciones de potasio y que la falta de nitrógeno afecta el rendimiento de tubérculo.

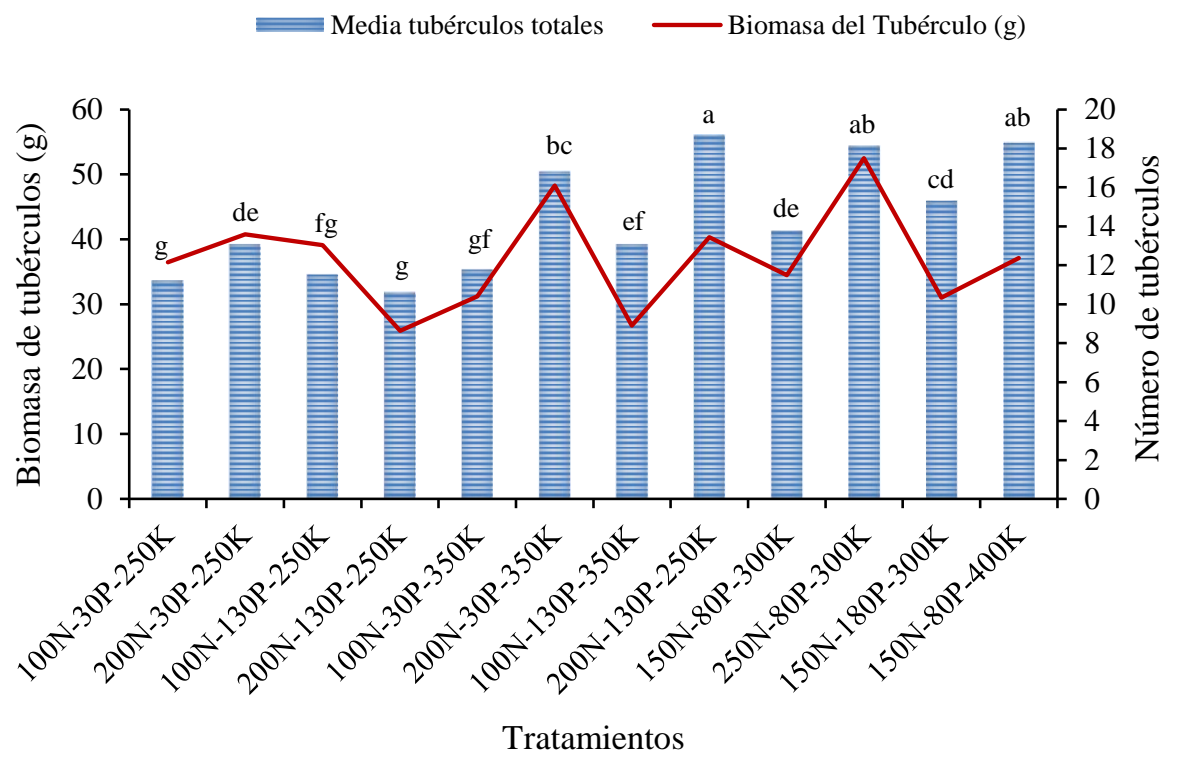

Figura 2. Comparación de número total de tubérculos y biomasa de tubérculos (g) producidos en invernadero de la variedad Nevada para el experimento de NPK en hidroponía. Tratamientos con la misma letra no son significativamente diferentes, Tukey $p<0.05$.

En relación al potasio, concuerda con Coraspe (2008); Moshileh y Errebi (2004) quienes mencionan que la aplicación de dosis crecientes de potasio en el cultivo de papa aumentaron la cantidad de biomasa producida por las plantas, requiriendo altas concentraciones de este para su crecimiento óptimo, producción y calidad del tubérculo, debido a que como se sabe el potasio activa enzimas necesarias para la producción de almidón y proteínas que ayuda a aumentar el peso de los granos y frutos (Rozo y Nústez, 2011).

En papa, Aguilar y Carrillo (2006), mencionan que antes de la tuberización los fotoasimilados se destinan principalmente para desarrollar hojas, tallos y raíces, y la fuerza de la demanda de las hojas es mayor que la de otro órgano; con el inicio de la tuberización esta tendencia cambia y a medida que los tubérculos crecen, su demanda por asimilados aumenta. Asimismo, se puede observar que los mejores tratamientos en peso seco de tubérculo contenían niveles de 200 y 250 $\mathrm{mg} \mathrm{L}^{-1}$ de nitrógeno, concuerda con lo mencionado por Sharifi et al. (2005) quienes localizaron, además que la absorción de la mayoría de $\mathrm{N}$ por la planta se produjo a los 76 días después de la emergencia; a partir, de entonces, la translocación de $\mathrm{N}$ del dosel a los tubérculos ocurre en respuesta a crecimiento del tubérculo.

Por otro lado, los tratamientos con mayor biomasa de hoja y tallo fueron los que tuvieron la mayor biomasa de tubérculo, contrario a Hancock et al. (2014), que señalan una menor acumulación de biomasa en hojas y tallos, asegura una mayor proporción hacia los tubérculos, esta afirmación se aplica en una nutrición balanceada, pues es de conocimiento que aplicaciones en exceso de nitrógeno alargan el ciclo de cultivo y disminuyen el rendimiento de papa (Rocha y Quijano, 2015). 
La mayor acumulación de biomasa en la planta completa se obtuvo en el tratamiento T10 (250 N; $80 \mathrm{P} ; 300 \mathrm{~K})$ con $63.5 \mathrm{~g}$ y T6 $(200 \mathrm{~N} ; 30 \mathrm{P} ; 350 \mathrm{~K})$ con $57.9 \mathrm{~g}$ y los tratamientos con menor biomasa total fueron T5 $(100 \mathrm{~N} ; 30 \mathrm{P} ; 350 \mathrm{~K})$ con $37.6 \mathrm{~g}$ y T7 $(100 \mathrm{~N} ; 130 \mathrm{P} ; 350 \mathrm{~K})$ con $34.5 \mathrm{~g}$. (Cuadro 2); la relación entre $\mathrm{N}$ y $\mathrm{K}$ es conocida desde hace tiempo respecto a la producción de biomasa en papa, concentraciones altas de nitrógeno, favorecen el desarrollo del dosel, alargan el ciclo de cultivo e inhiben la tuberización, mientras altas concentraciones de potasio la favorecen; sin embargo, se puede observar que a concentraciones de 200 a $250 \mathrm{de} \mathrm{N}$, se obtuvo mayor biomasa total y de tubérculo, lo que concuerda con Coraspe et al. (2008) quienes mencionan que la producción de biomasa está directamente relacionada con la dosis de nitrógeno en la solución nutritiva.

Cuadro 2. Acumulación de biomasa total e Índice de cosecha en papa con doce tratamientos de NPK en hidroponia e invernadero.

\begin{tabular}{ccc}
\hline Tratamientos & \multicolumn{2}{c}{ Biomasa $(\mathrm{g})$} \\
\cline { 2 - 3 } T1(100N-30P-250K) & $44.0 \mathrm{cebd}$ & Índice de cosecha \\
T2(200N-30P-250K) & $48.8 \mathrm{~b}$ & $0.82 \mathrm{ba}$ \\
T3(100N-130P-250K) & $47.3 \mathrm{~b}$ & $0.83 \mathrm{a}$ \\
T4(200N-130P-250K) & $32.5 \mathrm{e}$ & $0.82 \mathrm{ba}$ \\
T5(100N-30P-350K) & $37.6 \mathrm{ced}$ & $0.79 \mathrm{bc}$ \\
T6(200N-30P-350K) & $57.9 \mathrm{a}$ & $0.82 \mathrm{ba}$ \\
T7(100N-130P-350K) & $34.5 \mathrm{ed}$ & $0.82 \mathrm{ba}$ \\
T8(200N-130P-250K) & $48.8 \mathrm{~b}$ & $0.77 \mathrm{c}$ \\
T9(150N-80P-300K) & $42.8 \mathrm{cbd}$ & $0.82 \mathrm{ba}$ \\
T10(250N-80P-300K) & $63.5 \mathrm{a}$ & $0.8 \mathrm{bac}$ \\
T11(150N-180P-300K) & $39.9 \mathrm{cebd}$ & $0.82 \mathrm{ba}$ \\
T12(150N-80P-400K) & $46.1 \mathrm{cb}$ & $0.77 \mathrm{c}$ \\
DMS & 9.5 & $0.8 \mathrm{bac}$ \\
\hline
\end{tabular}

Tratamientos con la misma letra no difieren estadísticamente. Tukey $p<0.05$.

Por otro lado, la acumulación de biomasa total está determinada principalmente por el peso seco del tubérculo y no así por la biomasa de los demás órganos de la planta de papa.

En la variable de índice de cosecha existieron diferencias significativas entre tratamientos (Cuadro 2), este valor fluctuó de 0.77 a 0.83; sin embargo, nueve de los doce tratamientos fueron estadísticamente iguales, se puede observar también que un alto índice de cosecha no garantiza mayor eficiencia del cultivo, pues depende de la biomasa total, lo que indica es la distribución de biomasa en los diferentes órganos, como se observa en el tratamiento T2 (200 $\mathrm{N} ; 30 \mathrm{P} ; 250 \mathrm{~K}$ ) con el IC más alto de 0.83 , pero no con el mayor peso seco de tubérculo de $48.8 \mathrm{~g}$, en comparación con el T10 con biomasa de tubérculo de $52.5 \mathrm{~g}$ e IC de 0.82 igual estadísticamente al T2, mientras los tratamientos con un menor índice de cosecha fueron el T4 $(200 \mathrm{~N} ; 130 \mathrm{P} ; 250 \mathrm{~K})$, T7 (100 N; $130 \mathrm{P} ; 350 \mathrm{~K})$ y T11 (150N-180P-300K) con valores de 0.79 para el primero y 0.77 para los dos últimos. 
En general, estos resultados de IC superan a los obtenidos por Aguilar y Carrillo (2006), quienes en su investigación obtuvieron índices de cosecha de 69 y $62 \%$ en diferentes sistemas de riego. La relación que existe entre el peso seco total de la planta y el tubérculo es muy importante pues si se tiene un buen follaje, se incrementa la producción de fotoasimilados, los cuales al ser exportados a los órganos de interés económico contribuyen a incrementar el IC. Entre mayor sea el índice es más eficiente la planta para acumular materia seca en el órgano de interés.

En lo que se refiere al número de tubérculos totales (Figura 2) se observa diferencia significativa entre tratamientos debido posiblemente a las diferentes combinaciones de NPK empleadas. Los tratamientos con mayor producción de minitubérculos por planta fueron el T8 (200N-130P-250K) con 18.6 tubérculos, T12 (150N-80P-400K) 18.2, T10 (250N-80P-300K) 18.1 y T6 (200N-30P$350 \mathrm{~K}$ ) con 16.8, se observó que el $\mathrm{N}$ influyó en el número de tubérculos en tres de las cuatro soluciones, de las cuales su concentración fue igual o superior a $200 \mathrm{mg} \mathrm{L}^{-1}$; asimismo, el tratamiento con mayor número de minitubérculos fue el que presentaba $130 \mathrm{mg} \mathrm{L}^{-1}$ fósforo (T8), mientras el T12 con menor concentración de nitrógeno y alta de potasio con $400 \mathrm{mg} \mathrm{L}^{-1}$ fue igual estadísticamente a este último.

Estos resultados concuerdan con lo reportado por Alva et al. (2002); Giorgetta et al. (1993), quienes mencionan que el $\mathrm{N}$ es muy importante para el rendimiento de papa y que el $\mathrm{P}$ es importante en la generación de estolones y el rendimiento. El $\mathrm{N}$ es uno de los principales factores que incide en los rendimientos de la papa y se considera que la clave para aumentar el tamaño de los tubérculos, sin sacrificar su calidad, está en la aplicación adecuada de la fertilización nitrogenada (Ramírez et al., 2004).

Sin embargo, al observar la biomasa de tubérculos, solo los tratamientos T10 (250 N; $80 \mathrm{P} ; 300 \mathrm{~K})$ seguido del T6 $(200 \mathrm{~N} ; 30 \mathrm{P} ; 350 \mathrm{~K})$, presentaron valores altos de biomasa con 52.5 y $48.3 \mathrm{~g}$, mientras los tratamientos T12 y T8 presentaron una biomasa menor de 37.1 y $40.3 \mathrm{~g}$, respectivamente y mayor número de tubérculos (Figura 2); lo anterior, destaca la importancia de potasio y nitrógeno, en el T8(200N-130P-250K), falto potasio, pero la concentración de nitrógeno favoreció la tuberización, contrario al T12(150N-80P-400K) con alta concentración de potasio y bajo nitrógeno, esto indica la importancia de la disponibilidad de potasio en la solución desde el primer día del ciclo de cultivo, debido a que amarro gran cantidad de minituberculos, aunque la mayoría de menor diámetro y peso, lo que posiblemente se debió a la falta de nitrógeno en la solución pues este elemento que no sólo favorece la formación de hojas, sino es parte estructural de clorofila a y b que son las responsables de la fijación de carbono y en consecuencia producción de fotosintatos.

Resultados similares a los de esta investigación obtuvo Alva et al. (2002), en donde el peso seco del tubérculo representó de 76 a $87 \%$ del peso total de la planta. Los resultados mencionados indican la relación entre el nitrógeno y el potasio, que es determinante en la acumulación de peso seco de tubérculo. Ramírez et al. (2004) mencionan que el número de tubérculos por planta y la masa promedio por tubérculo se incrementó debido a la fertilización nitrogenada, oscilando entre 1.6 y $6.18 \mathrm{t} \mathrm{ha}^{-1}$, en forma proporcional con el aumento de la dosis del fertilizante nitrogenado hasta la aplicación de 100 y $150 \mathrm{~kg} \mathrm{ha}^{-1} \mathrm{de} \mathrm{N}$. 


\section{Conclusiones}

Altas concentraciones de nitrógeno y potasio en lasolución nutritiva favorecen mayorrendimiento en número y biomasa de minitubérculos, por lo que concentraciones mayores de $200 \mathrm{mg} \mathrm{L}^{-1}$ de nitrógeno y $300 \mathrm{mg} \mathrm{L}^{-1}$ de potasio en la solución nutritiva mostraron mejor producción de tubérculos y de biomasa en el cultivo de papa en hidroponía e invernadero.

La biomasa total está determinada principalmente por la biomasa del tubérculo y la mayor biomasa de la parte aérea (tallo y hojas) favorece mayor biomasa y número de minituberculos. Altas concentraciones de potasio aplicadas desde el inicio del ciclo de cultivo favorecen el amarre de minitubérculos durante el proceso de tuberización.

\section{Literatura citada}

Aguilar, A. J. L.; Martínez, H. J. J.; Volke, H. V.; Etchevers, B. J.; Sánchez, G. P. y Avendaño, S. R. 2001. Acumulación y distribución de la materia seca en papa cultivada con fertilización por goteo superficial y subsuperficial. Rev. Fitotec. Mex. 24(1):9-16.

Aguilar, M. y Carrillo, J. 2006. Análisis de crecimiento y relaciones fuente demanda de variedades de papa (Solanum tuberosum). Rev. Fitotec. Mex. 29(2):145-149.

Alva, K.; Hodges, T; Boydston, R. A. and Collins, H. P. 2002. Dry matter and nitrogen accumulations and partitioning in two potato cultivars. J. Plant Nutr. 25(8):1621-1630.

Becerra, S. y Ñústez, C. E. 2007. Efecto de niveles de fósforo y potasio sobre el rendimiento del cultiva "Criolla Guaneña". Rev. Latinoam. de la Papa. 14(1):51-60.

Bertsch, F. 2003. Absorción de nutrimentos por los cultivos. Asociación Costarricense de la ciencia del Suelo. San José, Costa Rica. 307 p.

Camozzi, M. E. 2015. Importancia y manejo del fósforo en cultivos hortícolas. Fertilcorp s.r.l Scrap de fertilizante orgánico y mineral. www.fertilcorp.com.

Chang, D. C.; Cho, I. C.; Suh, J.T.; Kim, S. J. and Lee, Y. B. 2011. Growth and yield response of three aeroponically grown potato cultivars (Solanum tuberosum L.) to different electrical conductivities of nutrient solution. Am. J. Potato Res. 88(6):450-458. http://dx.doi.org/10.1007/s12230-011-92116.

Chuquillanqui, C.; Mateus, J. y Otazu, V. 2010. Métodos de producción de semilla prebásica de papa. Centro Internacional de la papa (CIP). Apartado 1558, Lima, Perú. 1-2 p.

Coraspe, L. H. M.; Muroaka, T. I. y Do Prado, G. N. 2008. Nitrógeno y potasio en solución nutritiva para la producción de tubérculos- semilla de papa. Agron. Tropical. 58(4):417-425.

Flores, L. R.; Sánchez, F.; Rodríguez, E.; Colinas, T.; Mora R. y Lozoya, H. 2009. Densidad de población en el cultivo hidropónico para la producción de 56 tubérculos-semilla de papa (Solanum tuberosum L.). Rev. Chapingo Ser. Hortic. 15(3):251-258.

Giorgetta, B.; Dallari, P. y Buteler, M. 1993. Efectos de la fertilización fosforada sobre la producción de minituberculos de papa (Solanum tuberosum L.) en invernadero. Rev. Latinoam. de la Papa. 5(6):89-102.

Hancock, R. D.; Morris, W. L.; Ducreux, L. J. M.; Morris, J.A.; Usman, M.; Verrall, S. R.; Fuller, J.; Simpson, C. G.; Zhang, R.; Hedley, P. E. and Taylor, M. A. 2014. Physiological, biochemical and molecular responses of the potato (Solanum tuberosum L.) plant to moderately elevated temperature. Plant, Cell Environ. 37(2):439-450. 
Heemst, H. D. J. V. 1986. The distribution of dry matter during growth of a potato crop. Potato Res. 29(1):55-56.

Horneck, D. and Rosen, C. 2008. Measuring nutrients accumulation rates of potatoes too for better management. 92(1):1-4.

Jefferies, R. A. and Mackerron, K. L. 1993. Responses of potato genotypes to drought. II. Leaf area index, growth and yield. Ann. Appl. Biol. 122(1):105-112.

Khurana, S. C. and Pandita, M. L. 1994. Physiological innovations in potato production. J. Indian Potato Assoc. 21(1/2):47-52.

Martínez, G. A. 1996. Diseños experimentales. Editorial Trillas. México, DF. 756 p.

Mora, R.; Ortiz, J.; Rivera, A.; Mendoza, M. C., Colinas, M. T.; Lozoya H. y Rodríguez, J. E. 2005 Comportamiento de la acumulación y distribución de biomasa en genotipos de papa establecidos en condiciones de secano. Rev. Chapingo Ser. Hortic. 11(1):135-142.

Morales J. L; Hernández, J. y Rebollas, S. 2013. Rendimiento de papa con fuentes de fertilización mineral en un Andosol del Estado de México. Rev. Mex. Cienc. Agríc. 4(6):881-893.

Moshileh, A. M. and Errebi, M. A. 2004. Effect of various potassium sulfate rates on growth, yield and quality of potato grown under sandy soil and arid conditions. IPI Regional Workshop on Potassium and Fertirrigation Development in West Asia and North Africa. 24-28 pp.

Pumisacho, M. y Sherwood, S. 2002. El cultivo de la papa en Ecuador. Instituto Nacional Autónomo de Investigaciones Agropecuarias (INIAP)-Centro Internacional de la Papa (CIP). Quito. $231 \mathrm{p}$.

Rajwade, V. B.; Banafar, R. N. S. and Pathak, A. C. 2000. Growth analysis of potato in relation to biodinamic package and organic manures with chemical fertilizers. J. Indian Potato Assoc. 27(1/2):55-58.

Ramírez, O.; Cabrera, A. y Corbera, J. 2004. Fertilización nitrogenada de la papa (Solanum tuberosum L.) en la provincia de Holguín. Dosis óptima de nitrógeno. Rev. Científ. América Latina, el Caribe, España y Portugal. 25(2):75-80.

Rocha, R. y Quijano, J. A. 2015. Producción de papa en México. Instituto Nacional de Investigaciones Forestales, Agrícolas y Pecuarias (INIFAP). Centro de Investigación regional centro. Campo Experimental Bajío, Celaya, Gto. México. Folleto técnico núm. 1. $72 \mathrm{p}$.

Rolot, J. L. and Seutin, H. 1999. Soilless production of potato minitubers using a hydroponic technique. Potato Res. 42(3-4);457-469. http://dx.doi.org/ 10.1007/BF02358162.

Rozo, M. and Nústez, L. 2011. Effects of phosphorus and potassium levels on the yield of the tuber variety Criolla Colombia in the department of Cundinamarca. Agron. Colomb. 29(2):205- 212.

Sharifi, M.; Bernie, J. Z.; Hajabbasi, M. A. and Kalbasi, M. 2005. Dry Matter and nitrogen accumulation and root morphological characteristics of two clonal selections of russet Norkotah' potato as affected by nitrogen fertilization mehdi. J. Plant Nutr. 28(12):22432253.

Sierra, B. C.; Santos, R. J. y Kalazich, B. 2002. Manual de fertilización de cultivo de la papa en la zona Sur de Chile. Instituto de Investigaciones Agropecuarias. Boletín INIA N. 76. 104 p.

Susnoschi, M. and Shimshi, D. 1985. Growth and yield studies on potato development in an semiarid region. 2. Effect of water stress and amounts of nitrogen top dressing on growth of several cultivars. Potato Res. 28(2):161-176.

Valverde, F.; Córdova J. y Parra R. 1998. Fertilización del cultivo de papa. INIAP. Quito-Ecuador. $42 \mathrm{p}$.

Westermann, T. D. 2005. Nutritional requirements of potatoes. Am. J. Pot. Res. 82:301-307. 
White, P. J.; Whcatley, R. E; Hammond, J. P. and Zhang, K. 2007. Minerals, Soils and roots. In: Vreugdenhil, D. (Ed.). Potato biology and biotechnology, advances and perspectives. Elsevier Amtersdan. 739-752 p.

Wolf, S.; Marani, A. and Rudich, J. 1990. Effect of temperature and photoperiod on assimilate partitioning in potato plants. Ann. Bot. 66(5):513-520.

Zebarth, B. J. and Rosen, C. J. 2007 Research perspective on nitrogen BMP development for potato. Am. J. Potato Res. 84(1):3-18. 